\title{
Centre Vortices As The Origin Of Quark Confinement
}

\author{
Waseem Kamleh* \\ Special Research Centre for the Subatomic Structure of Matter, \\ Department of Physics, \\ University of Adelaide, Australia \\ E-mail: waseem.kamleh@adelaide.edu.au

\section{Derek B. Leinweber} \\ Special Research Centre for the Subatomic Structure of Matter, \\ Department of Physics, \\ University of Adelaide, Australia

\section{Daniel Trewartha} \\ Special Research Centre for the Subatomic Structure of Matter, \\ Department of Physics, \\ University of Adelaide, Australia
}

\begin{abstract}
Using lattice QCD, we examine several key features associated with dynamical chiral symmetry breaking and quark confinement. Calculations are performed in pure SU(3) gauge theory using the chiral overlap fermion action. Vortex-only and vortex-removed configurations are derived from the the original Monte Carlo gauge fields via a centre vortex identification procedure. The topological charge density, static quark potential, quark mass function and hadron spectrum are compared across the three ensembles. Remarkably, in each instance, the vortex-only degrees of freedom (after some smoothing) are able to encapsulate the pertinent features of the original gauge fields. The removal of centre vortices is consistently associated with a loss of dynamical chiral symmetry breaking. In particular, preliminary results for the vortex-removed meson spectrum hint at the restoration of chiral symmetry at light quark masses.
\end{abstract}

The 26th International Nuclear Physics Conference

11-16 September, 2016

Adelaide, Australia

${ }^{*}$ Speaker. 


\section{Introduction}

Quantum chromodynamics (QCD), the theory of the strong force, describes the interactions between quarks and gluons. The non-Abelian nature of QCD reflects the fact that the gluons are self-interacting, which in turn gives rise to a nontrivial vacuum state. This distinguishes the theory from quantum electrodynamics (QED), where perturbation theory upon a trivial vacuum has proved highly successful. In QCD, the nontrivial gluonic fluctuations present in the vacuum state yield a physical theory that is fundamentally non-perturbative at low energies. The nonperturbative nature of QCD is highlighted by the fact that the confinement of quarks inside hadrons and the dynamical generation of mass appear to be emergent properties, generally accepted to originate from the topological structure of the nontrivial vacuum. An analytic derivation of these phenomena remains elusive, however through the use of lattice gauge theory [1], a computational analysis of the properties of QCD becomes possible.

Lattice QCD discretises Euclidean space-time onto a four dimensional hypercube, placing the quark fields onto the vertices, and replacing the Hermitian gauge potential with the bi-local unitary gauge links $U_{\mu}(x)$ that serve as the parallel transporters between lattice sites. Equilibrium lattice gauge field configurations are generated via a Monte Carlo process and then sampled to evaluate path integrals. Normally we are interested in quantities averaged over an ensemble of Monte Carlo configurations, however it is also possible to learn something by examining the microscopic structures present in an individual representative gluon field configuration. In Figure 1, we display the topological charge density $q(x)$ calculated from the gluonic field strength tensor on a single timeslice. The Monte Carlo gauge field has been smoothed using 40 sweeps of cooling to filter out the high frequency fluctuations and reveal the underlying topological structures. Periodic boundary conditions are applied in all dimensions. The presence of roughly spherical, instanton-like topological objects is clear, a manifestation of the non-trivial QCD vacuum.

We seek a description of the essential nature of these topological structures and their connection to quark confinement. The centre vortex model $[2,3]$ is one candidate that has been explored extensively in lattice QCD, and here we review the recent work that has been performed by the

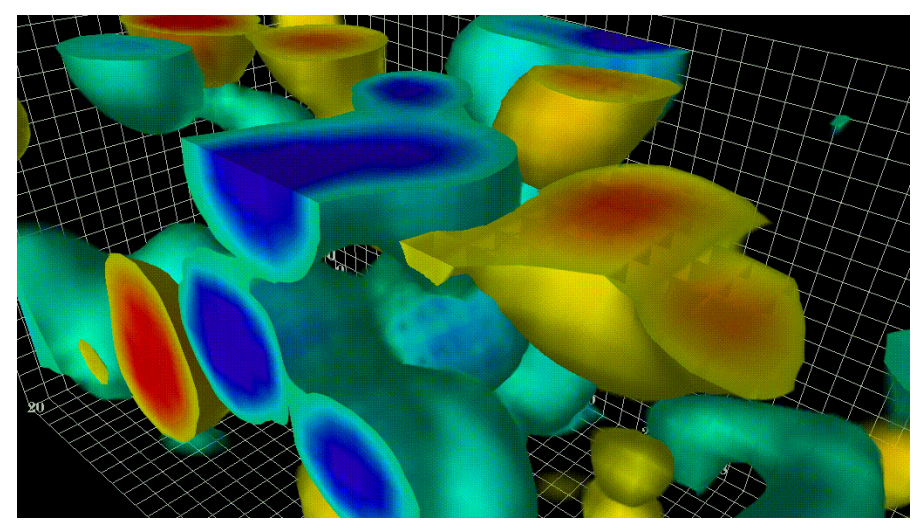

Figure 1: A visualisation of the topological charge density $q(x)$ after 40 sweeps of cooling applied to the gauge field $U_{\mu}(x)$ on a representative Monte Carlo configuration. Contours of positive topological charge density are plotted in yellow, and negative in blue. 
CSSM lattice collaboration $[4,5,6]$, and direct the reader therein for the details of the results presented here and a comprehensive list of references.

\section{Centre Vortices on the Lattice}

A singular gauge transformation, discontinuous on a 3-volume, defines a 2-dimensional centre vortex on the boundary. Specifically, a two-dimensional region $A$ of the gauge manifold $U$ intersects with a centre vortex if the Wilson loop identified with the boundary $\partial A$ has a nontrivial transformation property,

$$
U(\partial A) \underset{Z}{\rightarrow} z U(\partial A), \quad z \neq 1
$$

under an element $Z=z I \in \mathbb{Z}_{3}$ of the centre group of $\mathrm{SU}(3)$,

$$
Z=z \mathrm{I}, z \in\left\{1, e^{+2 \pi i / 3}, e^{-2 \pi i / 3}\right\}
$$

In the centre vortex model on the lattice, we seek to decompose the gauge links $U_{\mu}(x)$ in the form

$$
U_{\mu}(x)=Z_{\mu}(x) \cdot R_{\mu}(x),
$$

in such a way that all vortex information is captured in the field of centre-projected elements

$$
Z_{\mu}=\exp \left[\frac{2 \pi i}{3} m_{\mu}(x)\right] \mathrm{I}, \quad m_{\mu}(x) \in\{-1,0,1\}
$$

with the remaining short-range fluctuations described by the vortex-removed field $R_{\mu}(x)$. The field $Z_{\mu}(x)$ is constructed on the lattice by first fixing the gauge field to Maximal Centre Gauge and then projecting the gauge-fixed links to the nearest centre element. This centre-projected field is completely described by the integers $m_{\mu}(x)$, where each link has one of three values. The smallest closed loop on the lattice is the plaquette, the product of links around a $1 \times 1$ square. By searching the centre-projected field for plaquettes with a nontrivial centre flux around the boundary we can identify the vortex matter. These non-trivial plaquettes are the "thin" centre vortices, and are embedded within the physical "thick" centre vortices present in the original Monte Carlo configurations. Through the vortex identification procedure we create three distinct ensembles of SU(3) lattice gauge fields:

1. The original 'untouched' configurations, $U_{\mu}(x)$,

2. The projected vortex-only configurations, $Z_{\mu}(x)$,

3. The vortex-removed configurations, $R_{\mu}(x)=Z_{\mu}^{\dagger}(x) U_{\mu}(x)$.

Performing lattice calculations of key quantities on the three different ensembles and comparing the differences that emerge from the absence or presence of centre vortices provides revealing information regarding the properties of QCD. The results presented here are calculated within pure $S U$ (3) Yang-Mills theory.

Our first point of comparison between the three ensembles is at the microscopic scale through visualisations of the topological charge density. Figure 2 shows the topological charge density $q(x)$ on a single timeslice calculated from the vortex-only field $Z_{\mu}(x)$ (top) and vortex-removed field 


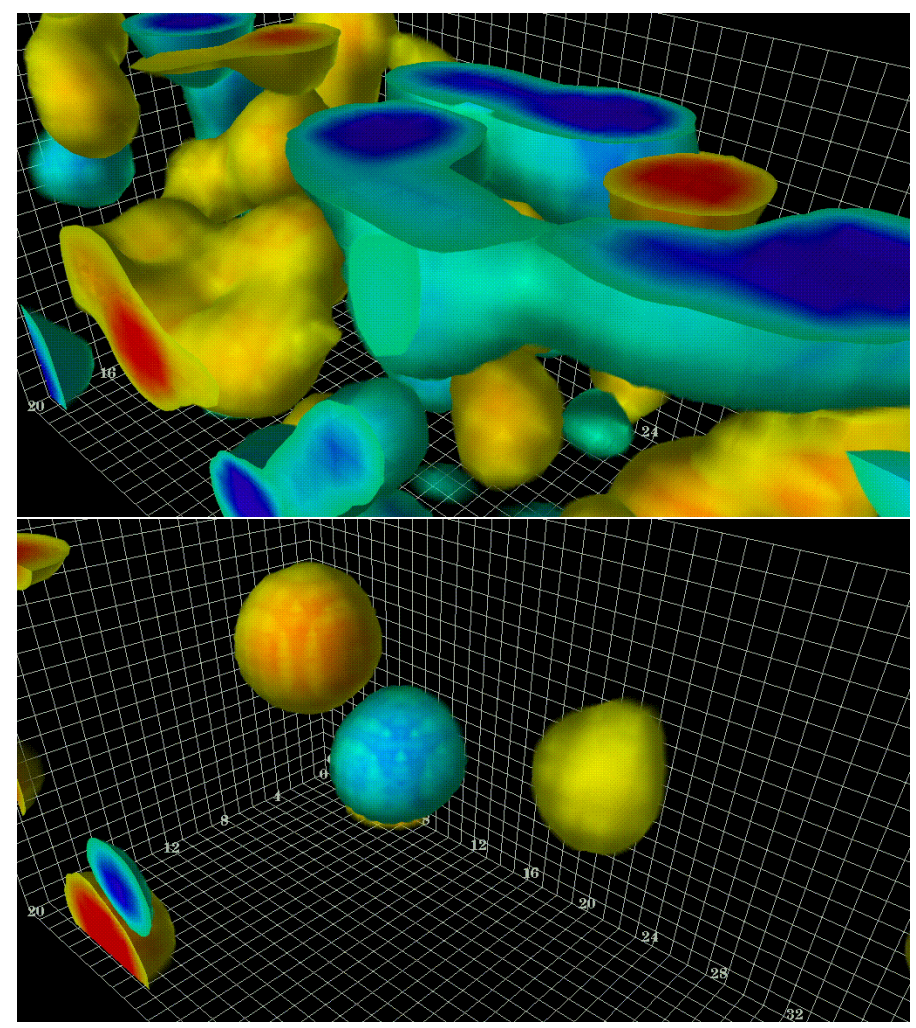

Figure 2: A visualisation of the topological charge density $q(x)$ after 40 sweeps of cooling applied the vortex-only field $Z_{\mu}(x)$ (top) and the vortex-removed field $R_{\mu}(x)$ (bottom) created from the same representative gauge field configuration as in Figure 1.

$R_{\mu}(x)$ (bottom) generated from the same untouched Monte Carlo configuration seen in Figure 1. Once again, after the centre projection procedure, 40 sweeps of cooling is applied to smooth away the short-range fluctuations present in order to reveal the long range physics. Indeed, without any cooling there is little or no visible difference in the topological charge density on the three ensembles. However, the application of cooling reveals some very interesting physics. The level of similarity between the untouched and vortex-only fields after 40 sweeps of cooling is striking. While the specific locations of the topological objects are different, the qualitative features of the untouched and vortex-only fields in terms of the strength and size of the topological objects are essentially identical. This presents an interesting concept, that the thin centre vortices are the seeds of the thick centre vortices, and through the application of cooling we may grow the thin vortices into fully-fledged, instanton-like topological objects. By contrast, after cooling the vortex-removed field is now almost entirely devoid of topology. There are some residual objects, associated with imperfections in the $S U(3)$ vortex-identification process which is known to be unable to simultaneously identify all vortex matter [7].

\section{Static Quark Potential}

Examining the topological charge density on individual configurations revealed a link between centre vortices and the instanton structure of the vacuum. We now turn our attention to quantities 


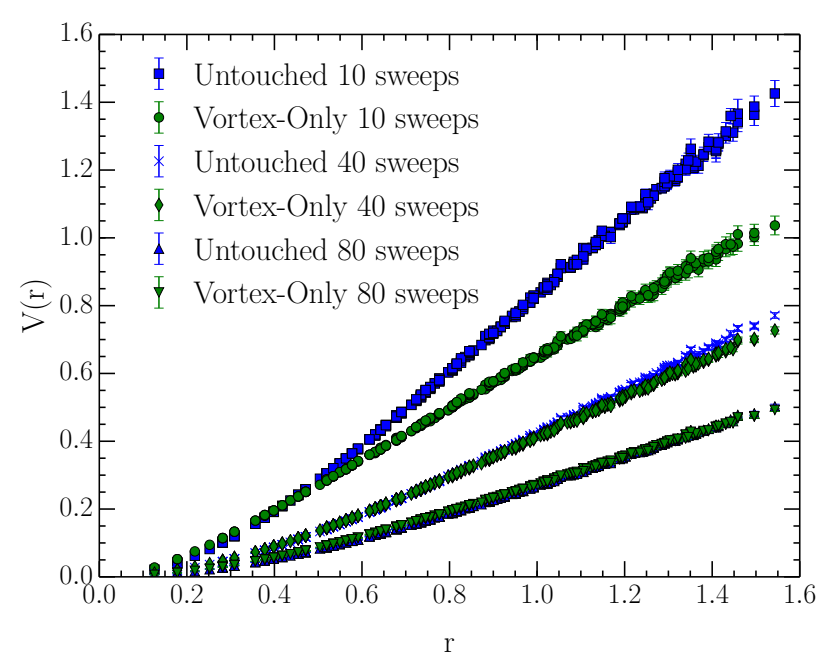

Figure 3: The static quark potential on untouched (blue) and vortex-only (green) configurations after 10, 40 , and 80 sweeps of cooling. Note that the 80 sweeps untouched potential is hidden behind the 80 sweeps vortex-only potential.

that can be measured at the ensemble level, commencing with the static quark potential. The connection between the existence of a linear potential, or string tension, and the notion of permanent quark confinement in pure Yang-Mills theory is well established [1,2]. This is the famous area law, where the string tension $\sigma$ relates the coefficient of the linear term in the static quark potential $V(r) \sim \sigma r$ to the exponential suppression of the Wilson loop by the area it encloses $W(\partial A) \sim e^{-\sigma A}$.

Previous results in $S U(3)$ lattice gauge theory show that while the removal of vortices results in the loss of the confining potential, on the vortex-only fields only about two-thirds of the linear potential is recovered [8]. The cause of this deficiency is understood to reside in the vortex identification procedure, which is imperfect. This imperfection was clearly visible in the previous section, where Fig. 2 showed that after cooling there were still topological objects present in the vortexremoved field $R_{\mu}(x)$. However, we see in Figure 3 that after only a small amount of cooling the vortex-only fields are able to recreate the string tension of the cooled untouched fields. While the identified centre vortices are inadequate to reproduce the full confinement potential, their evolution under smoothing is sufficient to match that of the cooled Monte Carlo fields. Future work will investigate finer lattice spacings under fixed iterations of cooling to learn if this is a lattice cutoff effect or a need to grow long-distance structures from the centre phases.

\section{Quark Mass Function}

The nontrivial nature of the QCD vacuum gives rise to dynamical chiral symmetry breaking, with the resulting non-perturbative quark-gluon interactions yielding the dynamical generation of mass that is responsible for $99 \%$ of the mass of the nucleon. In a covariant gauge, the lattice quark propagator can be decomposed into Dirac scalar and vector components as

$$
S(p)=\frac{Z(p)}{i \phi q+M(p)}
$$


where $M(p)$ is the non-perturbative quark mass function and $Z(p)$ is the renormalisation function. The infrared behaviour of the quark mass function $M(p)$ reveals the presence or absence of dynamical mass generation.

The left hand plot of Fig. 4 compares the quark mass function in Landau gauge on the untouched and vortex-removed ensembles (without any cooling). On the untouched ensemble, the mass function shows strong enhancement in the infrared, indicating the presence of dynamical mass generation. By contrast, dynamical mass generation is largely suppressed upon vortex removal with only a relatively small level of residual infrared enhancement remaining (again, likely to be associated with imperfections in the vortex-removal procedure). The removal of the vortex structure from gauge fields removes dynamical chiral symmetry breaking.

While many lattice fermion actions explicitly break chiral symmetry, overlap fermions obey the Ginsparg-Wilson relation [9]. This lattice-deformed version of chiral symmetry respected by the overlap operator is critical in revealing the subtle damage caused to the gauge fields through vortex removal. The overlap operator has a smoothness requirement $[10,11]$ for the underlying gauge field to preserve locality, and is not well-defined on the rough vortex-only fields consisting solely of elements from the centre group. However, the notion that the thin vortices are the seeds of the physical vortices motivates us to consider the vortex-only fields after cooling, which addresses the smoothness issue. The right hand plot of Fig. 4 compares the quark mass function on the untouched and vortex-only ensembles, both after 10 sweeps of cooling. The agreement between the two ensembles is remarkable, with the cooled vortex-only fields able to fully reproduce the dynamical mass generation present on the cooled Monte Carlo gauge fields.

\section{Hadron Spectrum}

The dynamical generation of mass is naturally connected to the hadron mass spectrum. The ability to reproduce the experimentally observed light hadron spectrum is a celebrated feature of lattice QCD. Noting the potential importance of chiral symmetry, we use overlap fermions to investigate what role centre vortices, and hence dynamical chiral symmetry breaking, play in the
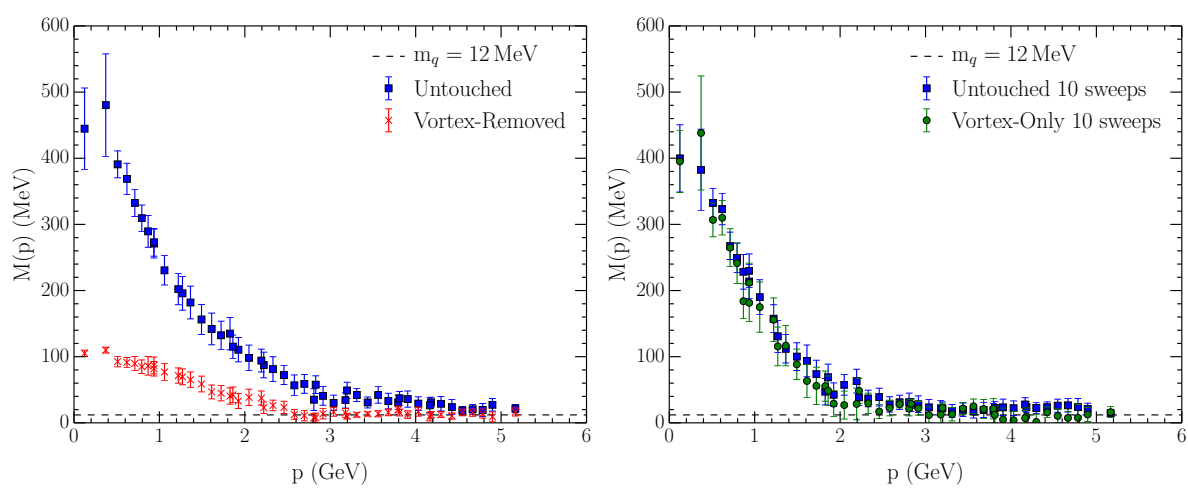

Figure 4: A comparison of the overlap quark mass function in Landau gauge at an input bare quark mass of $12 \mathrm{MeV}$ on the different ensembles. (Left) The mass function on the original (untouched) (squares) and vortex-removed (crosses) configurations. (Right) The mass function on the original (untouched) (squares) and vortex-only (circles) configurations after 10 sweeps of cooling. 
formation of the hadron spectrum. Figure 5 shows the effective mass plots for the light mesons $(\pi, \rho)$ and light baryons $(N, \Delta)$ calculated with overlap fermions on the untouched ensembles (with no cooling) and the vortex-only ensembles (with 10 sweeps of cooling to meet the overlap smoothness requirement). The cooled vortex-only ensemble is able to recreate the majority of the mass of the light hadrons, as well as the correct ordering. The vortex-only and untouched pion correlators are essentially identical, while for the $\rho, N$ and $\Delta$ the effective mass on the cooled vortex-only field is roughly $80 \%$ of the value on the original Monte Carlo gauge fields, in accord with other results [12].

Regarding the hadron spectrum on the vortex-removed ensemble, a previous study using Wilson-like fermions [4] (which explicitly break chiral symmetry) showed that the vortex-removed spectrum appeared to be described by weakly-interacting constituent quarks. Here we are using a chiral fermion action, so we are able to test if the removal of vortices results in the restoration of chiral symmetry. This leads to the postulation of two different scenarios in the vortex-removed spectrum. If the quark mass is large, chiral symmetry will be broken regardless. Hence, in the first scenario, at heavy pion masses we should observe a weakly interacting constituent quark regime, where the light hadron masses are simply a result of counting quarks. In this case, the $\pi$ and $\rho$ mesons should be degenerate. The $a_{0}$ and $a_{1}$ mesons should be degenerate with each other, but not with the $\pi$ or $\rho$ as due to parity considerations one of the constituent quarks must be excited with a single unit of momentum to create the appropriate quantum numbers.

At light quark masses the use of the overlap action should enable the restoration of chiral symmetry. The signal for this is that hadron currents related by the appropriate symmetries will become degenerate, while others remain distinct. In particular, the $a_{0}$ and $\pi$ should be degenerate, as should the $a_{1}$ and $\rho$ mesons, but the $\pi$ and $\rho$ should have different masses as they are not related by a chiral transformation. Figure 6 shows the effective mass functions for these mesons on the vortex-removed ensemble, in the chiral regime at $m_{q}=13 \mathrm{MeV}$ (left), and in the constituent regime at $m_{q}=126 \mathrm{MeV}$ (right). Indeed, we observe results that are consistent with our postulated scenarios (although we note that extracting the signal for the $a_{0}$ and $a_{1}$ mesons at the lightest quark mass is challenging). Further results for the vortex-removed mass spectrum with overlap fermions are presented elsewhere [13].
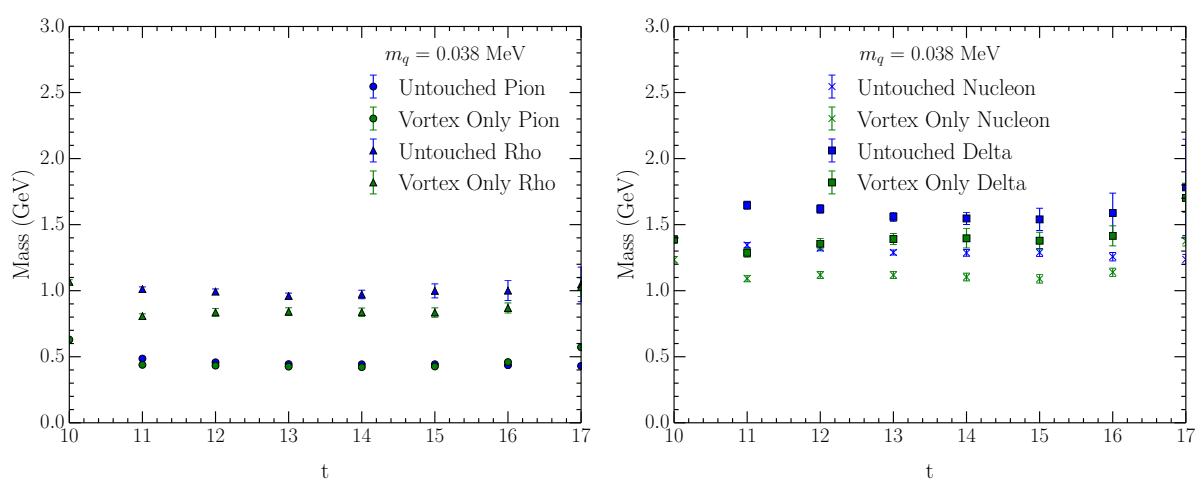

Figure 5: The effective masses of the low-lying mesons (left) and baryons (right) considered on the untouched (blue) and (10-sweep cooled) vortex-only (green) ensembles, at a bare quark mass of $38 \mathrm{MeV}$. 

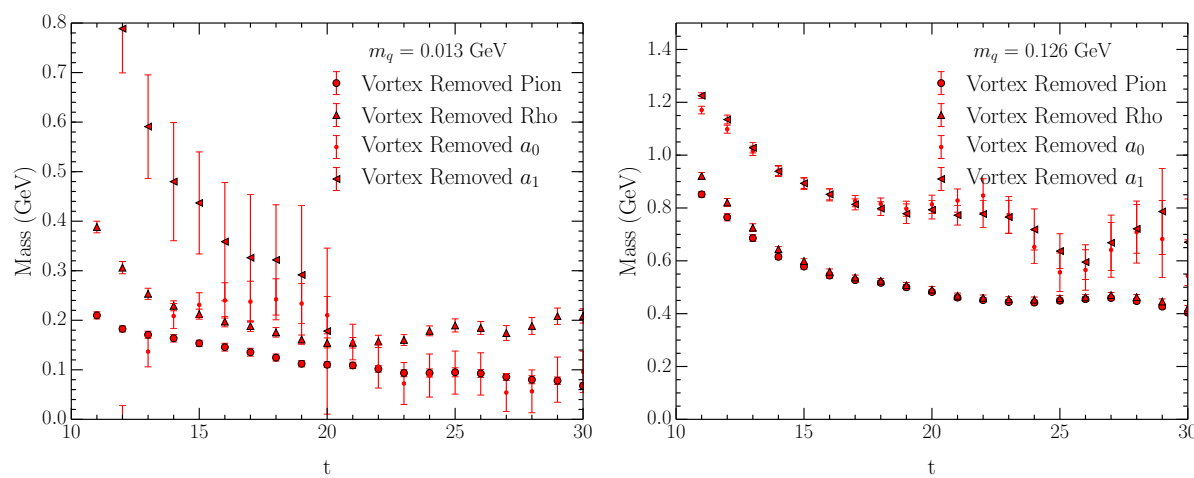

Figure 6: The effective mass functions of the $\pi, \rho, a_{0}$, and $a_{1}$ mesons on the vortex-removed ensemble, at a bare quark mass of $13 \mathrm{MeV}$ (left) and $126 \mathrm{MeV}$ (right).

In summary, we come to the remarkable conclusion that starting from the vortex-only fields, consisting only of centre elements, with the application of cooling we are able to reproduce all the salient features of QCD, including confinement and dynamical mass generation.

\section{Acknowledgements}

This research was undertaken with the assistance of resources at the NCI National Facility in Canberra, Australia, and the facilities at the Pawsey Supercomputing Centre in Western Australia, provided through the National Computational Merit Allocation Scheme and the University of Adelaide Partner Share. This research is supported by the Australian Research Council through grants DP150103164, DP120104627 and LE120100181.

\section{References}

[1] K. G. Wilson, Phys. Rev. D10 (1974) 2445-2459.

[2] G. 't Hooft, Nucl.Phys. B138 (1978) 1.

[3] G. 't Hooft, Nucl.Phys. B153 (1979) 141.

[4] E.-A. O'Malley et al., Phys.Rev. D86 (2012) 054503, [arXiv:1112 . 2490].

[5] D. Trewartha et al., Phys. Rev. D92 (2015), no. 7 074507, [arXiv: 1509.0551$].$

[6] D. Trewartha et al., Phys. Lett. B747 (2015) 373-377, [arXiv: 1502 . 0675].

[7] A. O'Cais et al., Phys.Rev. D82 (2010) 114512, [arXiv: 0807.0264 ].

[8] P. O. Bowman et al., Phys.Rev. D84 (2011) 034501, [arXiv: 1010 . 4624].

[9] H. Neuberger, Phys. Rev. D57 (1998) 5417-5433, [hep-lat/9710089].

[10] R. Narayanan and H. Neuberger, Nucl.Phys. B443 (1995) 305-385, [hep-th/ 9411108$].$

[11] R. Höllwieser et al., Phys.Rev. D78 (2008) 054508, [arXiv: 0805.1846 ].

[12] S. D. Thomas et al., Phys. Rev. D92 (2015), no. 9 094515, [arXiv: 1410 . 7105].

[13] D. Trewartha, W. Kamleh, and D. B. Leinweber, in preparation. 